\title{
SYNTHESIS, SPECTROSCOPIC CHARACTERIZATION AND X-RAY ANALYSIS OF 6-NITROQUINAZOLINE-2,4(1H,3H)-DIONE
}

\author{
VÍCTOR KESTERNICH ${ }^{*}$, MARCIA PÉREZ-FEHRMANN ${ }^{1}$, SERGIO ORTÍZ ${ }^{1}$, FELIPE VERDUGO , \\ IVÁN BRITO², MICHAEL BOLTE ${ }^{3}$ AND ALEJANDRO CÁRDENAS ${ }^{4}$ \\ ${ }^{1}$ Departamento de Química, Facultad de Ciencias, Universidad Católica del Norte, Av. Angamos 0610, Antofagasta, Chile. \\ ${ }^{2}$ Departamento de Quimica, Universidad de Antofagasta, Av.Angamos 601, Antofagasta, Chile \\ ${ }^{3}$ Institut für Anorganische Chemie, J. W. Goethe-Universität Frankfurt, Max-von-Laue-Strasse 7, 60438 Frankfurt/Main, Germany. \\ ${ }^{4}$ Departamento de Física, Universidad de Antofagasta, Av. Angamos 601, Antofagasta, Chile
}

(Received: March 14, 2013 - Accepted: May 7, 2013)

\begin{abstract}
In this work, 6-nitroquinazoline-2,4(1H,3H)-dione was obtained in two step with good yields using a facile synthetic method. Its structure was characterized by X-ray single crystal diffraction technique (XRD), IR, 1D-NMR ( ${ }^{1} \mathrm{H}-\mathrm{NMR}$ and $\left.{ }^{13} \mathrm{C}-\mathrm{NMR}\right)$ and 2D-NMR (H-H COSY and H-C HMBC) spectroscopy. All N and $\mathrm{O}$ carbonyl atoms are involved in hydrogen bonding, with an average $\mathrm{H} \cdots \mathrm{O}$ distance of $1.89(2) \AA$ and $\mathrm{N}-\mathrm{H} \cdots \mathrm{O}$ angles in the range $169.5(2)-177.2(2)^{\circ}$. In the crystal packing the molecules are associated by two strong intermolecular $\mathrm{N}-\mathrm{H} \cdots \mathrm{O}$ hydrogen bonds forming centrosymmetric ring with graph-set motif $\mathrm{R}_{2}^{2}(8)$, which are linked by $\mathrm{N}-\mathrm{H} \cdots \mathrm{O}$ hydrogen bonds.
\end{abstract}

\section{INTRODUCTION}

The quinazolinone nucleus and its derivatives have been extensively studied because of their wide range of pharmacological activities. As medicines, many of them display antifungal ${ }^{1}$, antimicrobial ${ }^{2}$, anti-HIV ${ }^{3}$, antitubercular ${ }^{4}$ , anticancer ${ }^{5}$, antiinflammatory ${ }^{6}$, anticonvulsant ${ }^{7}$, antidepressant ${ }^{8}$, hypolipidemic $^{9}$, antiulcer ${ }^{10}$, analgesic ${ }^{11}$ or immu-notropic activities ${ }^{12}$ and are also known to act as thymidyalate synthase ${ }^{13}$, poly(ADP-ribose) polymerase $(\mathrm{PARP})^{14}$, and protein tyrosine kinase ${ }^{15}$ inhibitors. As pesticides, they are used as insecticides ${ }^{16}$ and fungicides ${ }^{17}$. In light of the growing number of applications in recent years there has been an enormous increase in the interest among biologists and chemists in their synthesis and bioactivity of quinazoline derivatives. Several methods of synthesis quinazolindiones have been reported such as, from 2-aminobenzonitrile with supercritical $\mathrm{CO}_{2}{ }^{18}$, or by direct carbonylation of 2-aminobenzamide ${ }^{19}$ or starting from anthranilic acid with sodium cyanate, among others ${ }^{20}$. The structure has been confirmed by X-ray crystallography methods.

\section{RESULTS AND DISCUSSION}

Synthesis of 2,4-(1H,3H)-quinazolindione 3 (Scheme 1), was made it using the Lange y Sheible method ${ }^{20}$ modified, obtaining a better yield $(92 \%)$ respect to reported $(87 \%)$. The nitration was carried out using the procedure described by Mendenhall and Smith ${ }^{21}$ due they uses low temperatures and most favorable conditions for this type of reaction.<smiles>Nc1ccccc1C(=O)O</smiles>

1

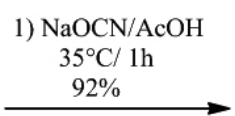

2) $\mathrm{H}_{2} \mathrm{SO}_{4} / \mathrm{HNO}_{3}$ $0^{\circ} \mathrm{C} / 3 \mathrm{~h}$ $82 \%$<smiles>[R]c1ccc2[nH]c(=O)[nH]c(=O)c2c1</smiles><smiles>Cc1c([N+](=O)[O-])cc2c(=O)n(C)c(=O)n(C)c2c1C</smiles><smiles>C1CCCCC2CC2CCC1</smiles>

b)

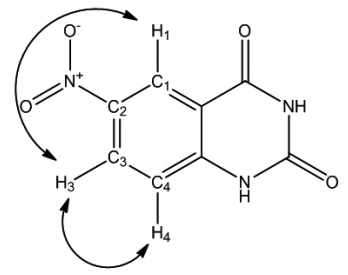

c)

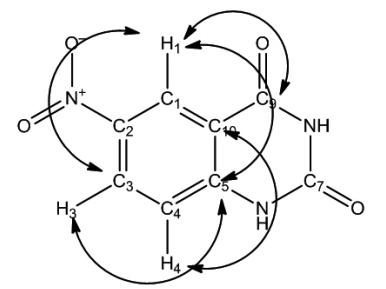

Figure 1: a) Numbering Scheme of 6-Nitro-2,4-(1H,3H)-quinazolindione, b) $\mathrm{H}-\mathrm{H}$ COSY and c) H-C HMBC principal correlations.

COSY experiment (Fig. 1b) of 3, we can see the interactions between $\mathrm{H} 1 /$ $\mathrm{H} 3$ and $\mathrm{H} 3 / \mathrm{H} 4$. HMBC spectrum shows correlations between $\mathrm{C} 3 / \mathrm{H} 1, \mathrm{C} 5 / \mathrm{H} 1$, $\mathrm{C} 9 / \mathrm{H} 1, \mathrm{C} 9 / \mathrm{H} 3$ and $\mathrm{C} 10 / \mathrm{H} 4$.

The crystal structure was determined by single crystal X-ray diffraction. The title compound is planar (rms deviation $0.074 \AA$ ), Figure 2 . All N and $\mathrm{O}$ carbonyl atoms are involved in hydrogen bonding, with an average $\mathrm{H} \cdots \mathrm{O}$ distance of $1.89(2) \AA$ and $\mathrm{N}-\mathrm{H} \cdots \mathrm{O}$ angles in the range $169.5(2)-177.2(2)^{\circ}$, so in the crystal packing the molecules are associated by two strong intermolecular $\mathrm{N}-\mathrm{H} \cdots \mathrm{O}$ hydrogen bonds forming centrosymmetric ring with graph-set motif ${ }^{22} \mathrm{R}_{2}{ }_{2}(8)$, which are linked by $\mathrm{N}-\mathrm{H} \cdots \mathrm{O}$ hydrogen bonds, Figure 3 , Table 2. $\pi-\pi$ stacking interactions are not observed. The bond length and angles are normal ${ }^{23}$.

Scheme1. Reagents and reaction conditions in synthesis of $\mathbf{3}$.

The complete ${ }^{1} \mathrm{H}$ and ${ }^{13} \mathrm{C}$ NMR assignments of compounds $\mathbf{3}$, based on one- and two-dimensional NMR experiments (e.g. Figure 1a; gives the atom numbering), is shown in Table 1. 
Table 1: ${ }^{1} \mathrm{H}$ and ${ }^{13} \mathrm{C}$ chemical shifts and 2D-NMR correlation data of compound 3 .

\begin{tabular}{|c|c|c|c|c|}
\hline Atom $\mathrm{N}^{\circ}$ & ${ }^{1} \mathrm{H} N M R$, multiplicity, $J(\mathrm{H}, \mathrm{H})(\mathrm{Hz})^{\mathrm{a}}$ & ${ }^{13} \mathrm{C}-\mathrm{APT} \mathrm{NMR}^{\mathrm{a}}$ & $\mathrm{COSY}^{\mathrm{b}}$ & $\mathrm{HMBC}^{\mathrm{c}}$ \\
\hline 1 & $8.59[\mathrm{H}-1, \mathrm{~d}, \mathrm{~J}(1,3)=2.62]$ & 123.06 & $1(3)$ & $1(\mathrm{C}: 2,3,5,9)$ \\
\hline 2 & - & 141.84 & - & - \\
\hline 3 & $8.45[\mathrm{H}-3, \mathrm{dd}, \mathrm{J}=9.01, \mathrm{~J}=2.66]$ & 129.55 & $3(1,4)$ & $3(\mathrm{C}: 1,2,5)$ \\
\hline 4 & $7.32[\mathrm{H}-4, \mathrm{~d}, \mathrm{~J}(4,3), \mathrm{J}=7.60]$ & 116.64 & $4(3)$ & $4(\mathrm{C}: 2,10)$ \\
\hline 5 & - & 145.61 & - & 1,3 \\
\hline 6 & $11.69[\mathrm{H}-6, \mathrm{~s}]$ & - & - & - \\
\hline 7 & - & 149.93 & - & - \\
\hline 8 & $11.74[\mathrm{H}-8, \mathrm{~s}]$ & - & - & - \\
\hline 9 & - & 161.57 & - & - \\
\hline 10 & - & 114.51 & - & - \\
\hline
\end{tabular}

${ }^{\mathrm{a}}$ In ppm from TMS; ${ }^{\mathrm{b}} \mathrm{H}-\mathrm{H} \mathrm{COSY}$ and ${ }^{\mathrm{c}} \mathrm{H}-\mathrm{C}$ HMBC interactions.

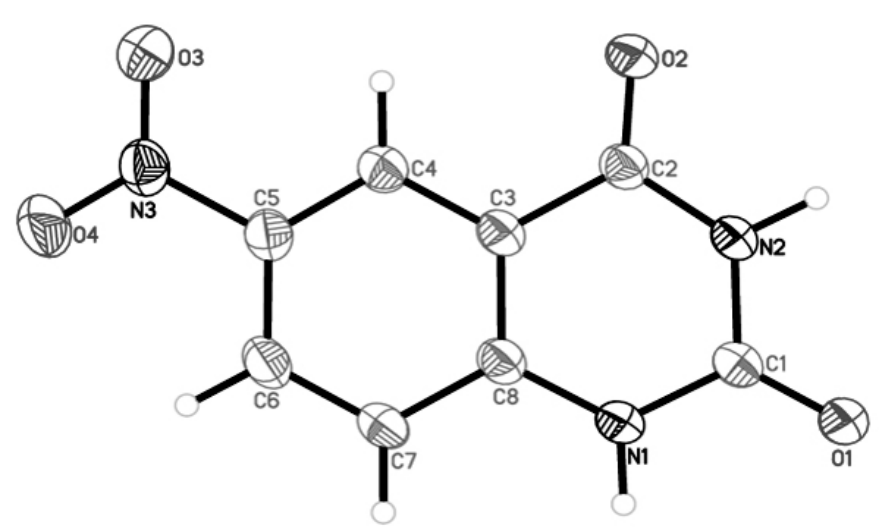

Figure 2.View of the title compound, showing 50\% probability displacement ellipsoids and atom-numbering scheme. $\mathrm{H}$ atoms are shown as small spheres of arbitrary radii.

Table 2. Hydrogen-bond geometry $\left(\AA,{ }^{\circ}\right)$.

\begin{tabular}{|l|c|c|c|c|}
\hline$D-\mathrm{H} \cdots A$ & $D-\mathrm{H}$ & $\mathrm{H} \cdots A$ & $D \cdots A$ & $D-\mathrm{H} \cdots A$ \\
\hline $\mathrm{N} 1-\mathrm{H} 1 \cdots \mathrm{O} 2^{\mathrm{ii}}$ & $0.887(18)$ & $1.974(18)$ & $\begin{array}{c}2.8512 \\
(14)\end{array}$ & $169.5(2)$ \\
\hline $\mathrm{N} 1-\mathrm{H} 2 \cdots \mathrm{O} 1^{\mathrm{i}}$ & $0.95(2)$ & $1.81(2)$ & $\begin{array}{c}2.7627 \\
(13)\end{array}$ & $177.2(2)$ \\
\hline
\end{tabular}

Symmetry codes: (ii) $-x,-y+2,-z+1$; (i) $x,-y+3 / 2, z+1 / 2$.

\section{EXPERIMENTAL}

Melting points were determined on a Kofler-type apparatus and are uncorrected. The IR were taken on a Perkin-Elmer 200 spectrophotometer with $\mathrm{KBr}$. NMR spectra were collected in DMSO-d6 or $\mathrm{CDCl}_{3}$ on a Varian Unity Inova $500 \mathrm{MHz}$ spectrometer equipped with a microflow probe from Protasis. Mass spectra were recorded on a Micromass-LCT Premier Time-of-Flight electrospray (ESI) spectrometer with interface system Acquity UPLC (Ultra Performance Liquid Chromatography). TLC was performed on Al Si gel Merck 60 F254 and TLC plates were visualized by spraying with phosphomolybdic acid reagent and heating. Commercially available, laboratory grade reagents were used without further purification.

\section{General Procedure}

Synthesis of quinazoline-2,4(1H,3H)-dione, 2

A solution containing $20 \mathrm{~g}(146 \mathrm{mmol})$ of anthranilic acid $\mathbf{1}$ in 0.71 of water and $11 \mathrm{~mL}(190 \mathrm{mmol})$ of acetic acid was heated at $35^{\circ} \mathrm{C}$ by 30 minutes. While the mixture is maintained stirring, a solution of $12 \mathrm{~g}(185 \mathrm{mmol})$ of sodium cyanate in $100 \mathrm{~mL}$ of water is dropwise slowly added and allowed to react for 30 minutes. After this time, the mixture was cooled on ice bath and after, $200 \mathrm{~g}$ ( 5 moles) of $\mathrm{NaOH}$ in small pieces were added slowly and stirring continued for 12 hours. The precipitate was washed in $1 \mathrm{~L}$ of water and neutralized with aqueous sulfuric acid (1:1) until $\mathrm{pH} 7$ and filtered under vacuum. The crude product, $21 \mathrm{~g}$ (yield 92\%) was crystallized in water giving colorless crystals (m.p. $350-351^{\circ} \mathrm{C}$ ).

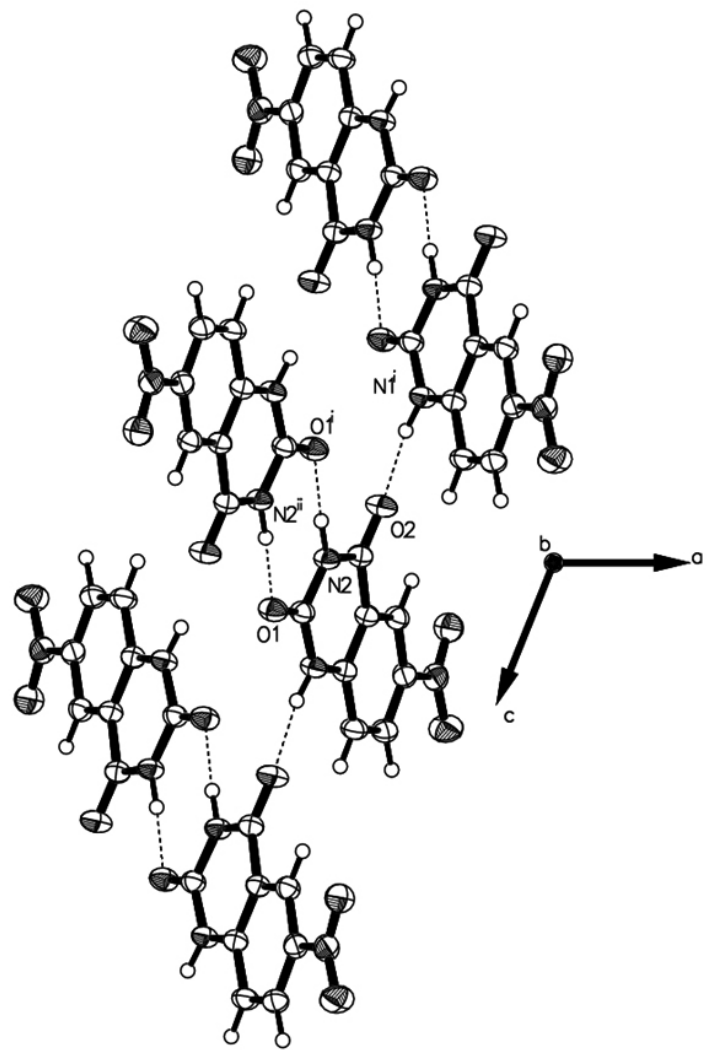

Figure 3. A view of the part of the crystal structure of the title compound showing $50 \%$ probability displacement ellipsoids illustrating the $\mathrm{N}-\mathrm{H} \cdots \mathrm{O}$ interactions. Dashed lines indicate hydrogen bonds. Symmetry codes: (ii) $-x$, $-y+2,-z+1$; (i) $x,-y+3 / 2, z+1 / 2$.

Spectroscopic data:

IR $\left(\mathrm{cm}^{-1}\right) 3254(\mathrm{~N}-\mathrm{H}), 3055(\mathrm{Csp} 2-\mathrm{H}), 1702(\mathrm{C}=\mathrm{O}), 1506$ (CAr-CAr).

${ }^{1} \mathrm{H}-\mathrm{NMR}$ (DMSO-d6, $\left.500 \mathrm{MHz}\right)(\mathrm{ppm}): 7.16(1 \mathrm{H}, \mathrm{m}, \mathrm{H} 4), 7.62(1 \mathrm{H}, \mathrm{t}$, $\mathrm{J}=7.7, \mathrm{H} 1), 7.88(1 \mathrm{H}, \mathrm{d}, \mathrm{J}=7.4, \mathrm{H} 3), 11.09(1 \mathrm{H}, \mathrm{s}, \mathrm{H} 6), 11.22(1 \mathrm{H}, \mathrm{s}, \mathrm{H} 8)$.

${ }^{13} \mathrm{C}-\mathrm{NMR}$ (DMSO-d6, $125.70 \mathrm{MHz}$ (ppm): 114.32 (C10), 115.28 (C2), 122.26 (C4), 126.91 (C1), 134.87 (C3), 140.83 (C5), 150.26 (C7), 162.79 (C9). 
HRESIMS: $\mathrm{m} / \mathrm{z} 163.0497$ for $\mathrm{C}_{8} \mathrm{H}_{7} \mathrm{~N}_{2} \mathrm{O}_{2}[\mathrm{M}+\mathrm{H}]^{+}$, calculated: $\mathrm{m} / \mathrm{z} 163.0508$. Synthesis of 6-Nitroquinazoline-2,4(1H,3H)-dione. 3

On an ice bath, $2 \mathrm{~g}(12,3 \mathrm{mmol})$ of quinazoline-2,4(1H,3H)-dione 2 , were dissolved in $19 \mathrm{~mL}$ of sulfuric acid. Maintaining the mixture under agitation, a solution of $0.68 \mathrm{~mL}$ of nitric acid $(12.3 \mathrm{mmol})$ and $1.37 \mathrm{~mL}$ of sulfuric acid were by dropwise added during 30 minutes and left reacting for 3 hours at $0^{\circ} \mathrm{C}$. After this time, the reaction mixture was poured into a beaker containing 70 $\mathrm{ml}$ cold water. Subsequently, $70 \mathrm{ml}$ of solution $9.5 \mathrm{~N}$ of $\mathrm{NaOH}$ was dropwise added. The resulting precipitate was filtered at vacuum, dried and purified by chromatographic column using petroleum-ether/ethylacetate (3/7) as eluent. This gave $2.1 \mathrm{~g}$ ( $82 \%$ yield) of a yellow product.

Spectroscopic Data:

IR $\left(\mathrm{cm}^{-1}\right) 3338(\mathrm{~N}-\mathrm{H}), 3018(\mathrm{Csp} 2-\mathrm{H}), 1683(\mathrm{C}=\mathrm{O}), 1533\left(\mathrm{CAr}-\mathrm{NO}_{2}\right)$.

${ }^{1} \mathrm{H}-\mathrm{NMR}$ (DMSO-d6, $500 \mathrm{MHz}$ (ppm): $7.32(1 \mathrm{H}, \mathrm{d}, \mathrm{J}=9, \mathrm{H} 4), 8.45(1 \mathrm{H}$, dd, J=9, H3), $8.59(1 \mathrm{H}, \mathrm{d}, \mathrm{J}=6.6, \mathrm{H} 1), 11.69(1 \mathrm{H}, \mathrm{s}, \mathrm{H} 6), 11.74(1 \mathrm{H}, \mathrm{s}, \mathrm{H} 8)$.

${ }^{13} \mathrm{C}-\mathrm{NMR}$ (DMSO-d6, $125.70 \mathrm{MHz}$ ) (ppm): 114.51 (C10), 116.64 (C4), 123.06 (C1), 141.84 (C2), 145.61 (C5), 149.93 (C7), 161.57 (C9).

HRESIMS: $\mathrm{m} / \mathrm{z} 206.0193$ for $\mathrm{C}_{8} \mathrm{H}_{4} \mathrm{~N}_{3} \mathrm{O}_{4}[\mathrm{M}-\mathrm{H}]^{+}$, calculated: $\mathrm{m} / \mathrm{z} 206.0202$. X- Ray Crystallography

Single crystal analysis data were collected on a Stoe IPDS-II two-circle Diffractometer with $\mathrm{MoK} \alpha$ radiation. Data collection: X-AREA ${ }^{24}$; cell refinement X-AREA ${ }^{24}$; data reduction: $\mathrm{X}_{\text {-AREA }}{ }^{24}$. Program used to solve structure program(s) used to refine structure: SHELXL9725; molecular graphics: XP in SHELXTL-Plus ${ }^{25}$ \& OLEX2 ${ }^{26}$, software used to prepare material for publication: SHELXL9725. Crystal dimension of $\mathrm{C}_{8} \mathrm{H}_{5} \mathrm{~N}_{3} \mathrm{O}_{4}, 0.37 \mathrm{x} 0.35 \mathrm{x} 0.12$ $\mathrm{mm}^{3} ; \mathrm{Mr}=207.15$ Dalton; Monoclinic P21/c; $\mathrm{a}=10.9071(8) \AA ; \mathrm{b}=6.5758(6)$ $\AA ; \mathrm{c}=12.4614(9) \AA ; \alpha=90^{\circ} ; \beta=112.382(5)^{\circ} ; \gamma=90.0^{\circ} ; \mathrm{V}=826.44(11) \AA^{3} ; \mathrm{Z}$ $=4 ; \mathrm{m}=0.137 \mathrm{~mm}^{-1} ; \mathrm{T}=173(2) \mathrm{K}$; absorption correction Semi-empirical from equivalents ${ }^{27-28}$; max. and min. transmission 0.9837 and 0.9509 . 8995/1898 measured/unique reflection with $\mathrm{I}>2 \mathrm{~s}(\mathrm{I})\left[\mathrm{R}_{\text {int }}=0.0556\right] ; \mathrm{R}[\mathrm{I}>2 \mathrm{~s}(\mathrm{I})] 0.0393, \mathrm{wR}_{2}$ $=0.1093 ; \mathrm{S}=1.097 ; 144$ parameters; $\operatorname{Dr}_{\text {max }}=0.284 \mathrm{e}^{-3}$ and $\operatorname{Dr}_{\text {min }}=-0.209 \mathrm{e}^{-3^{2}}$. $\mathrm{H} 1$ and $\mathrm{H} 2$ atoms were freely refined; all $\mathrm{H}$ atoms were located in difference maps and their positions and isotropic displacements parameters of 1.2 times those of the attached atoms. Supplementary information: Crystallographic data (excluding structure factors) for the structural analysis have been deposited in the Cambridge Crystallographic Data Centre, CCDC 927348. These data can be obtained free of charge from the Cambridge Crystallographic Data Centre; Postal Address : CCDC, 12 Union Road, Cambridge CB21EZ, UK, Telephone: (44) 01223 762910, Fax: (44) 01223 336033, e-mail: deposit@ccdc.cam.ac.uk

\section{REFERENCES}

1. J. Bartroli, E. Turmo, M. Alguero, E. Boncompte, M.L. Vericant, J. Conte Ramis, M. Merlos, J.F. Gracia-Rafanell. J. Med. Chem. 48 (1998) 18691882 .

2. I.M.Sh. El-Sharief, Y.A. Ammar, M.A. Zahran, A.H. Ali, M.S.A. ElGaby, Molecules 6 (2001) 267-278.

3. D.M. Purohit, V.R. Bhuva, V.H. Shah, An Indian Journal 1 (2003) 233245.

4. K.M. Murav'eva, N.V. Arkhangel'skaya, M.N. Shchukina, T.N. Zykova, G.N. Pershin, Khim. Farm. Zh. 5 (1971) 25-27.

5. S.G. Abdel-Hamid, J. Indian Chem. Soc. 74 (1997) 613-618.

6. A.J. Barker, Eur. Pat 635498, 1995 [Chem. Abstr. 122 (1995) 214099].

7. M.A. Aziza, M.K. Ibrahim, A.G. El-Helpy, Al-Azhar J. Pharm. Sci. 14 (1994) 193-201.

8. N. Ergenc, S. Buyuktimkin, G. Capan, G. Baktir, S. Rollas, Pharmazie 46 (1991) 290-291.

9. A.A. Bekhit, M.A. Khalil, Pharmazie 53 (1998) 539-544

10. E. Hamel, C.M. Lin, J. Plowman, H.K. Wang, K.H. Lee, K.D. Paull. Biochem. Pharmacol. 51 (1996) 53-59.

11. K. Terashima, H. Shimamura, A. Kawase, Y. Tanaka, T. Tanimura, T. Kamisaki, Y. Ishizuka, M. Sato. Chem. Pharm. Bull. 43 (1995) 2021-2023.

12. A. Gursoy, N. Karali, Farmaco 50 (1995) 857-866.

13. D.J. Baek, Y.K. Park, H.I. Heo, M.H. Lee, Z.Y. Yang, M.H. Chio, Bioorg. Med. Chem. Lett. 8 (1998) 3287-3290.

14. R.J. Grif fi n, S. Srinivasan, K. Bowman, A.H. Calvert, N.J. Curtin, D.R. Neweli, L.C. Pemberton, B.T. Golding, J. Med. Chem. 41 (1998) 52475256.

15. B. Sumegi, K. Hideg, T. Kalai. US Pat. Appl. 20070042935, 2007.

16. M. Uehara, T. Shimizu, S. Fujioka, M. Kimura, K. Tsubata, A. Seo. Pestic. Sci. 55 (1999) 359-362.

17. G.P. Ouyang, P.Q. Zhang, G.F. Xu, B.A. Song, S. Yang, L.H. Jin, W. Xue, D.Y. Hu, P. Lu, Z. Chen. Molecules 11 (2006) 383-392.
18. T. Mizuno, T. Iwai, Y. Ishino; Tetrahedron Lett. 45 (2005) 7073-7075.

19. T. Miyata, T. Mizuno, Y. Nagahama, I. Nishiguchi, T. Hirashima, N. Sonoda,; Heteroat. Chem, 2 (1991) 473-475.

20. N. A. Lange and F. E. Sheibley. Org. Synt. 2 (1943) 79.

21. G. D. Mendenhall, P. Smith. Org. Synt. 46 (1966) 85.

22. J. Bernstein, R.E. Davis, L. Shimoni, \& N.-L. Chang, Angew. Chem. Int. Ed. Engl. 34 (1995) 1555-1573.

23. F.H. Allen, O. Kennard, D.G. Watson, L. Brammer, A.G. Orpen \& R. Taylor. J. Chem. Soc. Perkin Trans. 2 (1987) S1-19.

24. Stoe \& Cie (2001). X-AREA. Stoe \& Cie, Darmstadt, Germany.

25. G.M. Sheldrick, Acta Cryst. A64 (2008) 112-122.

26. O.V. Dolomanov, L.J. Bourhis, R.J. Gildea, J.A.K. Howard, H. Puschmann. OLEX2: A complete structure solution, refinement and analysis program. J. Appl. Cryst. 42 (2009) 339-341.

27. A. L. Spek, Acta Cryst. D65 (2009) 148-155.

28. R. H. Blessing, Acta Cryst. A51 (1995) 33-38. 